\title{
Numerical simulation of Gate shape effect on Self- Heating in nano-MOSFET Transistors with high-k dielectric
}

\section{Maissa Belkheria}

Universite de Monastir Faculte des Sciences de Monastir

Fraj Echouchene

Universite de Monastir Faculte des Sciences de Monastir

Abdullah Bajahzar

Majmaah University

hafedh belmabrouk ( $\nabla$ ha.belmabrouk@mu.edu.sa )

Majmaah University https://orcid.org/0000-0003-0840-0271

\section{Research Article}

Keywords: Gate shape, MOSFET, Finite element method, Numerical simulation, Self-heating effect (SHE)

Posted Date: April 14th, 2021

DOI: https://doi.org/10.21203/rs.3.rs-370785/v1

License: (c) This work is licensed under a Creative Commons Attribution 4.0 International License.

Read Full License 


\title{
Numerical simulation of Gate shape effect on Self-Heating in nano-MOSFET Transistors with high-k dielectric
}

\author{
Maissa Belkhiria ${ }^{(1)}$, Fraj Echouchene ${ }^{(1)}$, Abdullah Bajahzar $^{(2)}$, Hafedh Belmabrouk $^{\left(1,3,{ }^{*}\right)}$ \\ ${ }^{(1)}$ Laboratory of Electronics and Microelectronics, University of Monastir, 5000, Tunisia \\ Computer Science and Information, College of Science at Zulfi, Majmaah University, Saudi Arabia \\ ${ }^{(2)}$ Department of Physics, College of Science at Zulfi, Majmaah University, Saudi Arabia \\ *Corresponding author: Ha.Belmabrouk@mu.edu.sa
}

\begin{abstract}
:
The aim of the present work is to investigate numerically the self-heating effect (SHE) in MOSFET transistors based on high-k material taking into account the deformation of the gate under the SHE. The SHE inside the MOSFET transistor is calculated using the electrothermal model based on heat transfer equation coupled with semiconductor equations. The electrothermal model have been solved in 2D-dimension using the finite element method. The high-k dielectric $\mathrm{HfO}_{2}$ have been used as gate oxide. Several gate shapes have been used to analyze their impact on SHE. It is observed that the reduction of equivalent oxide thickness (EOT) reduces the SHE in the MOSFET transistor based in high-k dielectric material. the temperature peak increases quadratically with drain voltage for all MOSFET structures. A decrease in self-heating effect is achieved using the square gate shape.
\end{abstract}

Key words: Gate shape, MOSFET, Finite element method, Numerical simulation, Self-heating effect (SHE).

\section{Introduction:}

The intensive scaling of MOSFET transistors requires the thinning of the $\mathrm{SiO}_{2}$ gate oxide which induces significant gate tunnels leading to power loss, increased power consumption, and generation of excess heat [1-6]. The increase of drain current caused by the low dissipation capacity of the dioxide $\mathrm{SiO}_{2}$ [7] results an increase in self-heating effect (SHE) [8]. Therefore, using highk oxides than $\mathrm{SiO}_{2}$ oxide can mitigate this issue. In our previous work [9], we have proved that the 
fact of using a high-k material able to reduce the device temperature and then to mitigate the SHE. Otherwise, we have investigated the effect of different high-k materials such as $\mathrm{ZrO}_{2}, \mathrm{HfO}_{2}, \mathrm{La}_{2} \mathrm{O}_{3}$, and $\mathrm{Al}_{2} \mathrm{O}_{3}$ on SHE in PiFETs Structures [10]. An important reduction of SHE was obtained using these dielectric materials. Some other studies have demonstrated a decrease of gate tunneling current is obtained by using the high-k dielectric materials [11]. According to Yu et al.[12], a good enhancement in transfer and output characteristics is achieved in their experimental study of strained SiGe quantum well p-MOSFETs with higher-k dielectric. Evenly, Sharma et al. [13] have shown that the use of high $\mathrm{k}$ materials in JL-CSG MOSFET device contributes to the improvement of the electrical and thermal characteristics.

Pravin et al. [14] have studied the dual metal gate MOSFET transistor with with different h-k dielectric materials. The impact of high-k gate dielectric on electrical characteristics have been simulated in Karbalaei et al.[15] work. They have investigated the electric performance with varying angle of coverage in a circular cross-section of gate-all-around field-effect transistor (GAAFET). The simulation results revealed that the electrical control of the gate over the channel increase as $\mathrm{HfO}_{2}$ cover more the channel.

In order to study the self-heating effect (SHE) in nano electronic components, several electrothermal models have been developed and used [5, 6, 9, 16-18]. Belkhiria et al. [16] have analyzed the SHE in the gate-all-around-Field-Effect Transistor (GAAFET) using on the Cattaneo and Vernotte (CV) heat conduction model. Echouchene et al.[5, 6] have investigated the entropy generation in MOSFET transistor using the dual-phase-lag (DPL) model. They have shown that the entropy generation cannot be described using the classical form of the equilibrium entropy production and an oscillatory behavior in transient entropy generation obtained using DPL model in a real MOSFET transistor.

In the other hand, the effect of gate shape on electrical and self-heating characteristics in nanowire transistor have been investigate by $[19,20]$.

In this paper we propose to study the gate shape effect on self-heating in MOSFET transistors based on $\mathrm{HfO}_{2}$ as high-k gate dielectric instead of $\mathrm{SiO}_{2}$. Several gate shapes have been used in this analysis such as curve, square and triangular gate shape. The results have been compared to conventional MOSFET structure with plane gate shape. In this investigation, the continuity and 
Poisson equations coupled with the heat transfer equation have been solved based on the finite element method.

\section{Electrothermal model for MOSFET transistor}

\subsection{Device structure}

Figure 1 presents the geometry of typical transistor studied in this work. The thin layer of $\mathrm{SiO}_{2}$ insulator is placed above the channel and another layer of high-k dielectric located between the dioxide and the gate.

The high-k gate oxides are physically thicker layers than $\mathrm{SiO}_{2}$. This will retain the same capacity but decreases the tunnel current. Therefore, it is appropriate to define the equivalent oxide thickness (EOT) which is defined the $\mathrm{SiO}_{2}$ layer thickness that would be required to achieve the same capacitance density as the high-k material:

$E O T=\left(\frac{3.9}{\varepsilon_{H . K}}\right) \times t_{H . K}$

where 3.9 is the dielectric constant of the oxide, $\varepsilon_{H . K}$ is the dielectric constant for high-k material, and $t_{H . K}$ is its thickness. The geometric parameters are illustrated in Table $\mathbf{1}$.

\subsection{Electrothermal model}

The electrothermal model used in this work is given by [10]:

$$
\left\{\begin{array}{l}
\nabla \cdot(\varepsilon \nabla V)+q(p-n)=q\left(N_{A}-N_{D}\right) \\
\frac{\partial p}{\partial t}+\nabla \cdot\left(p \vec{v}_{p}\right)=\nabla \cdot\left(D_{p} \nabla p\right)-R_{p} \\
\frac{\partial n}{\partial t}+\nabla \cdot\left(n \vec{v}_{n}\right)=-\nabla \cdot\left(D_{n} \nabla n\right)-R_{n} \\
C \frac{\partial T_{L}}{\partial t}=\nabla \cdot\left(\lambda \nabla T_{L}\right)+H
\end{array}\right.
$$

where $V, n, p$ and $T_{L}$ are respectively, the electrostatic potential, the electron concentration, the hole concentration and the lattice temperature, $N_{D}$ and $N_{A}$ are the ionized donor and acceptor impurity concentrations, respectively. $D_{n, \mathrm{p}}$ present the diffusion coefficients of electron and hole, respectively. $\lambda$ is the thermal conductivity, $C$ is the specific heat, $\varepsilon$ is the local permittivity, $q$, is the magnitude of the charge on an electron, $R_{n}$ and $R_{p}$ are the electron and hole recombination rates, 
respectively. $H=\vec{J} \cdot \vec{E}$ is the heat generation rate, where $E$ is the built-in electric field, $J$ is the electric current density in the active zone.

\subsection{Numerical method}

The numerical solution of the transport equation (Eq.2) has been done using a finite element method. The approximation of function $\Phi=\left[V, p, n, T_{L}\right]$ can be expanded in terms of the shape function as:

$\Phi(x, y, t)=\sum_{i=1}^{N} \Phi_{i}(t) \phi_{i}(x, y)$

In order to proceed we introduce a complete finite set of shape functions $\phi_{i}(x, y): j=1,2, \ldots, N$. The integral form is obtained by multiplying (2) by $\phi_{j}$ and integrating over the region $\Omega$ occupied by the device. After applying the divergence theorem we find :

$$
\left\{\begin{array}{l}
\int_{\Omega} \nabla \phi_{j}(\varepsilon \nabla V) \partial \Omega-\int_{\Omega} \phi_{j} q\left(p-n+N_{A}-N_{D}\right) \partial \Omega=\int_{\Gamma} \phi_{j}(\varepsilon \nabla V) \partial \Gamma \\
\int_{\Omega} \phi_{j} \frac{\partial p}{\partial t} \partial \Omega+\int_{\Omega} \nabla \phi_{j}\left(D_{p} \nabla p-p \vec{v}_{p}\right) \partial \Omega+\int_{\Omega} \phi_{j} R_{p} \partial \Omega=\int_{\Gamma} \phi_{j}\left(D_{p} \nabla p-p \vec{v}_{p}\right) \partial \Gamma \\
\int_{\Omega} \phi_{j} \frac{\partial n}{\partial t} \partial \Omega-\int_{\Omega} \nabla \phi_{j}\left(D_{n} \nabla n+n \vec{v}_{n}\right) \partial \Omega+\int_{\Omega} \phi_{j} R_{n} \partial \Omega=-\int_{\Gamma} \phi_{j}\left(D_{n} \nabla n+n \vec{v}_{n}\right) \partial \Gamma \\
\int_{\Omega} \phi_{j} C \frac{\partial T_{L}}{\partial t} \partial \Omega+\int_{\Omega} \nabla \phi_{j}\left(\lambda \nabla T_{L}\right) \partial \Omega-\int_{\Omega} \phi_{j} H \partial \Omega=-\int_{\Gamma} \phi_{j}\left(\lambda \nabla T_{L}\right) \partial \Gamma
\end{array}\right.
$$

The left terms of Eq. 4 represent the boundaries conditions make the integrals over $\Gamma$ vanish. After developpement, Eq. 4 can be written as:

$\sum_{i}\left[\alpha M_{i j} \dot{\Phi}_{i}+\left(\beta K_{i j}+\gamma L_{i j}\right) \Phi_{i}\right]+F_{j}=0 \quad 1 \leq j \leq M$

where

$$
\begin{aligned}
& M_{i j}=\int_{\Omega} \phi_{i} \phi_{j} \partial \Omega, \quad L_{i j}=\int_{\Omega} \phi_{i} \nabla \phi_{j} \vec{v}_{\Phi} \partial \Omega, \\
& K_{i j}=\int_{\Omega} \nabla \phi_{i} \nabla \phi_{j} \partial \Omega \text { and } F_{j}=\int_{\Omega} \delta_{\Phi} \phi_{j} \partial \Omega
\end{aligned}
$$

and 
$\alpha=\left(\begin{array}{l}0 \\ 1 \\ 1 \\ C\end{array}\right), \beta=\left(\begin{array}{c}0 \\ D_{p} \\ -D_{n} \\ \lambda\end{array}\right), \gamma=\left(\begin{array}{c}0 \\ -1 \\ -1 \\ 0\end{array}\right), \delta_{\Phi}=\left(\begin{array}{l}q / \varepsilon\left(N_{D}-N_{A}\right) \\ R \\ R \\ -H\end{array}\right)$

After assembling the elementary matrices, we obtain the global matrix form:

$[M] \alpha \dot{\Phi}+(\beta[K]+\gamma[L]) \Phi+[F]=0$

where $\Phi$ is the vector of unknown nodal transportable quantity, $[M]$ is the damping matrix, $(\beta[K]+\gamma[L])$ is the stiffness matrix, and $F$ is the external flux vector.

The discretization of the ordinary differential equation Eq.8 gives

$\Phi_{n+1}=\Phi_{n-1}-\frac{2 \Delta t}{\alpha M}\left[(\beta[K]+\gamma[L]) \Phi_{n}+[F]\right]$

where $n$ is the time index and $\Delta t$ is time discretisation step.

To carry out the thermal analysis of the proposed structures of MOSFET transistor, we adopted the following strategy for solving this problem:

- Poisson and continuity equations are solved iteratively, with a convergence achieved.

- Heat transfer equation is solved with an initial temperature $300 \mathrm{~K}$ assumed for the device so as to predict the local temperature.

The numerical code was validate with Rahiman and al.[21] results in a SOI MOSFET structure.

Figure 2 presents $I_{D}-V_{D}$ characteristics compared to the experimental data [21].

\section{Results and discussion}

Gate shape effect's on the electro-thermal behavior have been investigated in MOSFET structure based on high-k material. $\mathrm{HfO}_{2}$ has been chosen as a gate dielectric placed above the dioxide $\mathrm{SiO}_{2}$ with an equivalent oxide thickness (EOT).

Figure 3 depicts the temperature distribution along $X-Y$ plane for $V_{D}=2 \mathrm{~V}$ and $V_{G}=1.2 \mathrm{~V}$. The results are calculated in MOSFET structure as shown in figure 1. The dielectric gate $\mathrm{HfO}_{2}$ is placed above the dioxide $\mathrm{SiO}_{2}$ with EOT. As it can be seen from figure 3, the temperature maximal 
especially in the $\mathrm{SiO}_{2}-\mathrm{Si}$ interface due to the low thermal conductivity of $\mathrm{SiO}_{2}$ layer. The temperature decreases until the substrate temperature which is similar to the previous work [10].

Figure 4 present the electric potential distribution in MOSFET transistor for $V_{G}=1.2 \mathrm{~V}$ and $V_{D}=2$ V. It's clear that the maximum value of electric potential is in the drain part.

The dielectric thickness effect on the SHE in MOSFET structure is studied for different EOT and for a variable drain voltage as shown in figure 5 at fixed $V_{G}=1.2 \mathrm{~V}$. The simulation results suggest that the highest temperature is obtained with the high value of $\mathrm{V}_{\mathrm{D}}$ and the low of EOT. However, the peak temperature value proves that at this point the tunneling probability of the carrier to the gate terminal through the oxide is maximum. Therefore, we should choose the low EOT value in order to significantly reducing the impact ionization.

Subsequently, we propose to study the gate shape effect three different gate shape as shown in figure 1 (b-d) are chosen in order to analyze the SHE in these structures. Figure 6 shows the maximum temperature variation with the drain voltage for a constant $\mathrm{V}_{\mathrm{G}}=1.2 \mathrm{~V}$. This study exhibits a quadratic temperature increase with the drain voltage $V_{D}$. It is clear from this figure that the gate shape affects the temperature variation. The lowest temperature is achieved with square gate shape. For example, for $\mathrm{V}_{\mathrm{D}}=2 \mathrm{~V}$ the maximum temperature is $352.89 \mathrm{~K}, 323.94 \mathrm{~K}$ and $322.5 \mathrm{~K}$ in $\mathrm{T}$ MOSFET, C-MOSFET and S-MOSFET, respectively.

Figure 7 shows the variation of the temperature profile along the y axis in the centerline at the hot spot location near the drain region. The results are shown for $\mathrm{V}_{\mathrm{D}}=2 \mathrm{~V}$ and $\mathrm{V}_{\mathrm{G}}=1.2 \mathrm{~V}$. The peak temperature is located at $\mathrm{y}=0$ in the $\mathrm{Si}-\mathrm{SiO}_{2}$ interface. This maximum decreases significantly up to the substrate temperature $\mathrm{T}=300 \mathrm{~K}$ and the temperature have the same trend in all devices with different gate shape.

The temperature profile along the $\mathrm{x}$ direction is presented in figure $\mathbf{8}$ at the $\mathrm{Si}-\mathrm{SiO}_{2}$ interface. It is clear from this figure that the SHE is located in the drain side channel for all structures. Also, the hot spot is located near the drain region and the maximum temperature reaches up to $325 \mathrm{~K}$ using the triangular gate shape. The results obtained with the S-MOSFET are slightly higher than TMOSFET and C-MOSFET. It is found that the use of square gate shape can endure the increase of the temperature in nanodevices. 
The transient temperature profile in normal MOSFET is shown in figure 9 for different drain voltage. The results show the maximum temperature, which is located near the drain region at the $\mathrm{Si} / \mathrm{SiO}_{2}$ interface for $\mathrm{V}_{\mathrm{G}}=1.2 \mathrm{~V}$. The temperature profile increases rapidly for $\mathrm{t}<100 \mathrm{ps}$ and slightly for $\mathrm{t}>100 \mathrm{ps}$ until the steady state is reached at $\mathrm{t}=500 \mathrm{ps}$ for different $\mathrm{V}_{\mathrm{D}}$. The same study has been made in C-MOSFET, S-MOSFET and T-MOSFET structures with the same values of $V_{D}$ and for the same $\mathrm{V}_{\mathrm{G}}$. The relevant results show the same form of temporal temperature evolution, obtained with different structures, with different amplitude. For examples, for $\mathrm{V}_{\mathrm{D}}=2 \mathrm{~V}$ and at $\mathrm{t}=500$ ps, $\mathrm{T}_{\max }=321 \mathrm{~K}, 319 \mathrm{~K}, 318 \mathrm{~K}$ and $322 \mathrm{~K}$ in normal MOSFET, C-MOSFET, S-MOSFET, and TMOSFET, respectively. Furthermore, we noted from these figures that the steady state is reached at $\mathrm{t}=500 \mathrm{ps}$ for the C-MOSFET, while it is at $\mathrm{t}=700 \mathrm{ps}$ for the S-MOSFET and the T-MOSFET.

Figure 10 shows the comparison of the temporal evolution of the peak temperature rise in different devices for constant $\mathrm{V}_{\mathrm{D}}=2 \mathrm{~V}$ and $\mathrm{V}_{\mathrm{G}}=1.2 \mathrm{~V}$. Our results are compared with BTE and BDE models. It is clear from this figure that the steady state in T-MOSFET structure is achieved earlier than the other structures. However, a significant temperature rise is obtained using the BTE and BDE models and the temperature achieve so fast the steady state.

\section{Conclusion}

On this work, the self-heating effect is analyzed in MOSFET transistor with different gate shapes. The heat transfer equation and the semiconductor equations have been used to investigate the temperature distribution along devices. The effect of drain voltage and EOT have been discussed. In addition, the special and temporal evolution have been analyzed. The obtained results show that using different gate shape able to mitigate the SHE. Especially, the square gate shape is more appropriate to reduce the temperature device.

\section{Declarations}

Funding: Not applicable

Conflicts of interest/Competing interests: No conflict of interest

Availability of data and material (data transparency): All data related to this publication are made available in the article. 
Code availability: Not applicable

Authors' contributions: All co-authors contributed in all parts of the manuscript.

Ethics approval: Not applicable. The study does not involve humans or animals.

Consent to participate: Not applicable. The study does not involve humans or animals.

Consent for publication: Not applicable

\section{References}

1. Koh, M., et al., Limit of gate oxide thickness scaling in MOSFETs due to apparent threshold voltage fluctuation induced by tunnel leakage current. IEEE Transactions on Electron Devices, 2001. 48(2): p. 259-264.

2. Pimbley, J.M. and J.D. Meindl, MOSFET scaling limits determined by subthreshold conduction. IEEE transactions on electron devices, 1989. 36(9): p. 1711-1721.

3. Seo, K.-I., et al. A $10 \mathrm{~nm}$ platform technology for low power and high performance application featuring FINFET devices with multi workfunction gate stack on bulk and SOI. in 2014 Symposium on VLSI Technology (VLSI-Technology): Digest of Technical Papers. 2014. IEEE.

4. Zeitzoff, P.M. MOSFET scaling trends and challenges through the end of the roadmap. in Proceedings of the IEEE 2004 Custom Integrated Circuits Conference (IEEE Cat. No. 04CH37571). 2004. IEEE.

5. Echouchene, F. and H. Belmabrouk, Effect of Temperature Jump on Nonequilibrium Entropy Generation in a MOSFET Transistor Using Dual-Phase-Lagging Model. Journal of Heat Transfer, 2017. 139(12).

6. Echouchene, F. and H.B. Mabrouk. Non equilibrium entropy generation in nano scale MOSFET transistor based a nonlinear DPL heat conduction model. in 20189 th International Renewable Energy Congress (IREC). 2018. IEEE.

7. Cheng, M.-C., et al., Steady-state and dynamic thermal models for heat flow analysis of silicon-on-insulator MOSFETs. Microelectronics Reliability, 2004. 44(3): p. 381-396.

8. Fiegna, C., et al., Analysis of self-heating effects in ultrathin-body SOI MOSFETs by device simulation. IEEE Transactions on Electron Devices, 2007. 55(1): p. 233-244.

9. Belkhiria, M., F. Echouchene, and H. Mejri. Nanoscale heat transfer in MOSFET transistor with high-k dielectrics using a non linear DPL heat conduction model. in 2018 9th International Renewable Energy Congress (IREC). 2018. IEEE.

10. Belkhiria, M., et al., Impact of High-k Gate Dielectric on Self-Heating Effects in PiFETs Structure. IEEE Transactions on Electron Devices, 2020. 67(9): p. 3522-3529.

11. Cheng, B., et al., The impact of high-/spl kappa/gate dielectrics and metal gate electrodes on sub-100 nm MOSFETs. IEEE Transactions on Electron Devices, 1999. 46(7): p. 15371544.

12. Yu, W., et al., High mobility compressive strained Si0. 5Ge0. 5 quantum well p-MOSFETs with higher-k/metal-gate. Solid-state electronics, 2011. 62(1): p. 185-188. 
13. Sharma, A., et al., Effect of high-k and vacuum dielectrics as gate stack on a junctionless cylindrical surrounding gate (JL-CSG) MOSFET. Solid-State Electronics, 2016. 123: p. 26-32.

14. Pravin, J.C., et al., Implementation of nanoscale circuits using dual metal gate engineered nanowire MOSFET with high-k dielectrics for low power applications. Physica E: Lowdimensional systems and nanostructures, 2016. 83: p. 95-100.

15. Karbalaei, M., D. Dideban, and H. Heidari, Impact of high-k gate dielectric with different angles of coverage on the electrical characteristics of gate-all-around field effect transistor: A simulation study. Results in Physics, 2020. 16: p. 102823.

16. Belkhiria, M., et al., 2-D-Nonlinear Electrothermal Model for Investigating the SelfHeating Effect in GAAFET Transistors. IEEE Transactions on Electron Devices, 2021.

17. Echouchene, F. and E. Jemii, Analysis of the transient Joule heating effect in a conductivebridge random-access memory (CBRAM) using a single-phase-lag (SPL) model. Journal of Computational Electronics, 2021: p. 1-8.

18. Gazzah, M.H., et al., Electro-thermal modeling for InxGa1-xN/GaN based quantum well heterostructures. Materials Science in Semiconductor Processing, 2019. 93: p. 231-237.

19. Song, Y.S., et al., Electrical and Thermal Performances of Omega-Shaped-Gate Nanowire Field Effect Transistors for Low Power Operation. Journal of nanoscience and nanotechnology, 2020. 20(7): p. 4092-4096.

20. Wang, W., Z. Liu, and T.-K. Chiang, A new effective-conducting-path-driven subthreshold behavior model for junctionless dual-material omega-gate Nano-MOSFETs. IEEE Transactions on Nanotechnology, 2019. 18: p. 904-910.

21. Rahimian, M. and A.A. Orouji, A novel nanoscale MOSFET with modified buried layer for improving of AC performance and self-heating effect. Materials science in semiconductor processing, 2012. 15(4): p. 445-454. 


\section{$\underline{\text { Figure captions }}$}

Figure 1: 2D Normal MOSFET (a), MOSFET with curve gate shape (b), MOSFET with square gate shape (c) and MOSFET with triangular gate shape (d)

Figure 2 : Comparison of the output caractesistics of simulated results with exprimental data[21]

Figure 3 : Temperature distribution along X-Y plane

Figure 4 : Electric potential distribution along X-Y plane

Figure 5 : Temperature distribution for different EOT and variable drain voltage

Figure 6 : Maximum temperature profile versus drain voltage in MOSFET structure with different gate shape

Figure 7 : Temperature profile along the y axis in MOSFET structure with different gate shape

Figure 8 : Temperature profile along the $\mathrm{x}$ axis in MOSFET structure with different gate shape

Figure 9 : Transient temperature profile in MOSFETs for different drain voltages.

Figure 10 : Comparaison of Transient temperature profile in different structures 


\section{Table captions}

Table 1: Device parameters of MOSFET transistor 
Figure 1

(a)

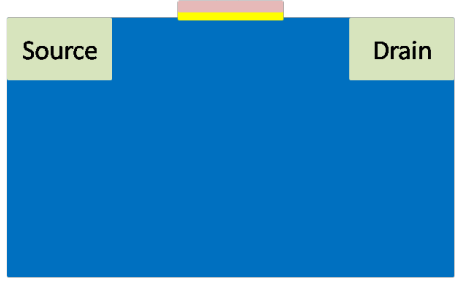

(c)

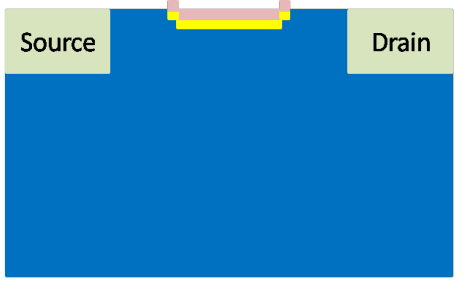

(b)

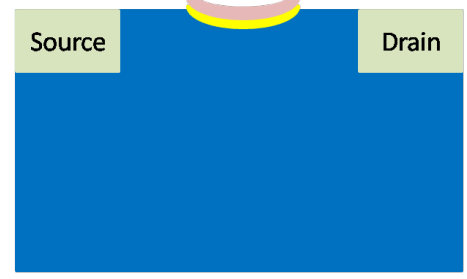

(d)

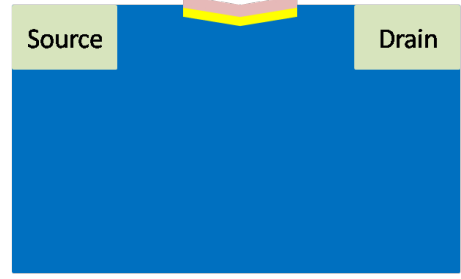


Figure 2

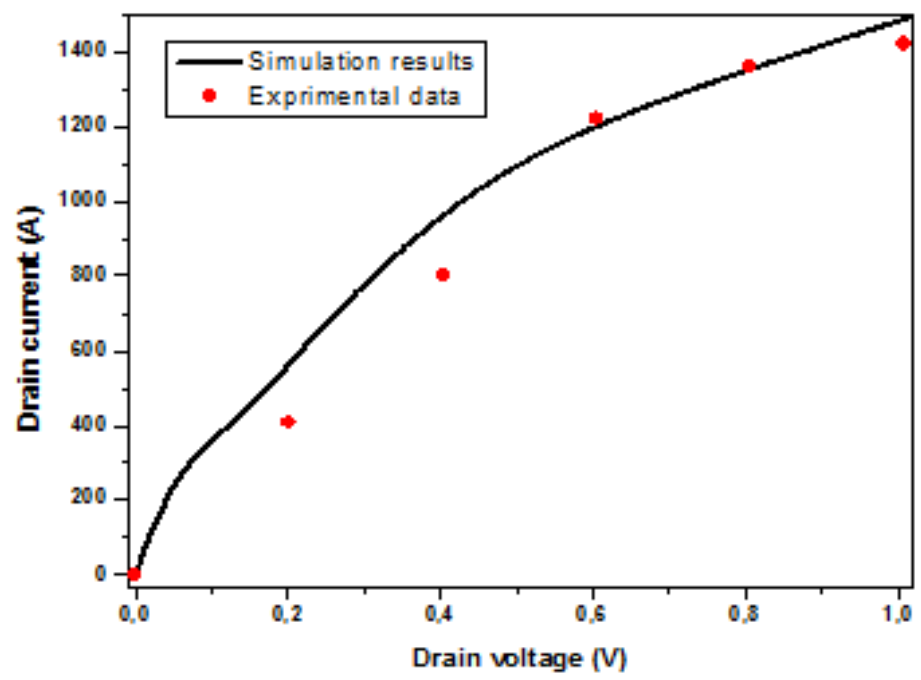


Figure 3

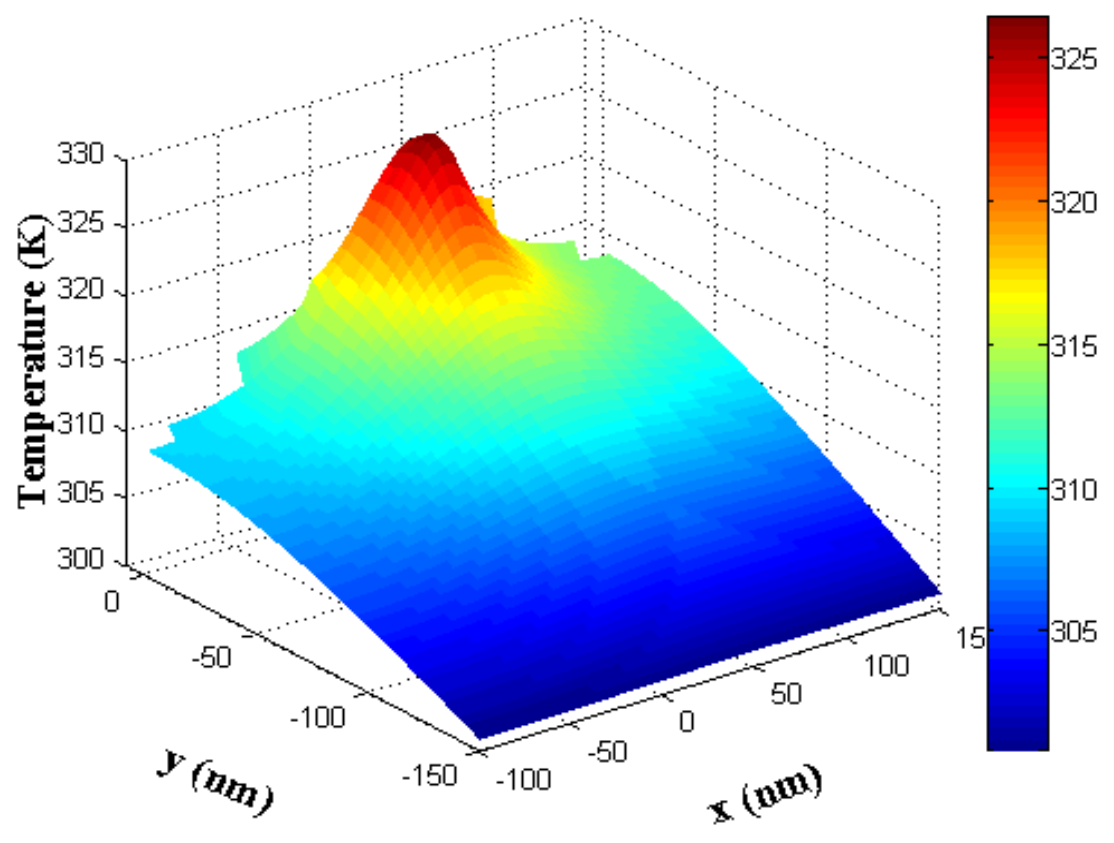


Figure 4

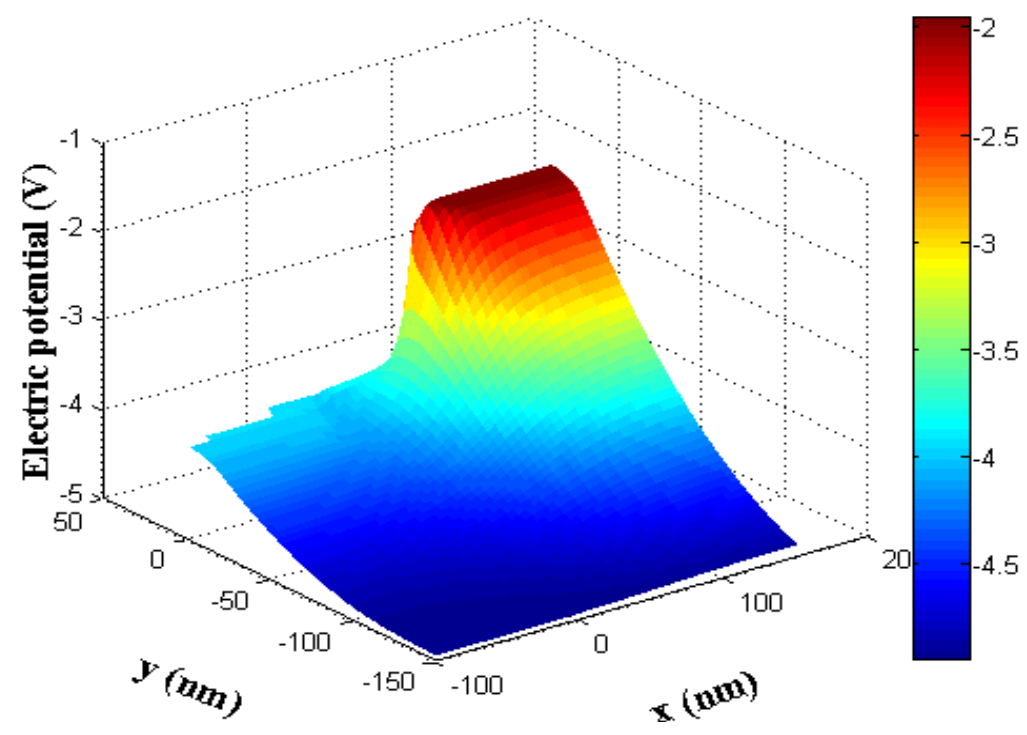


Figure 5

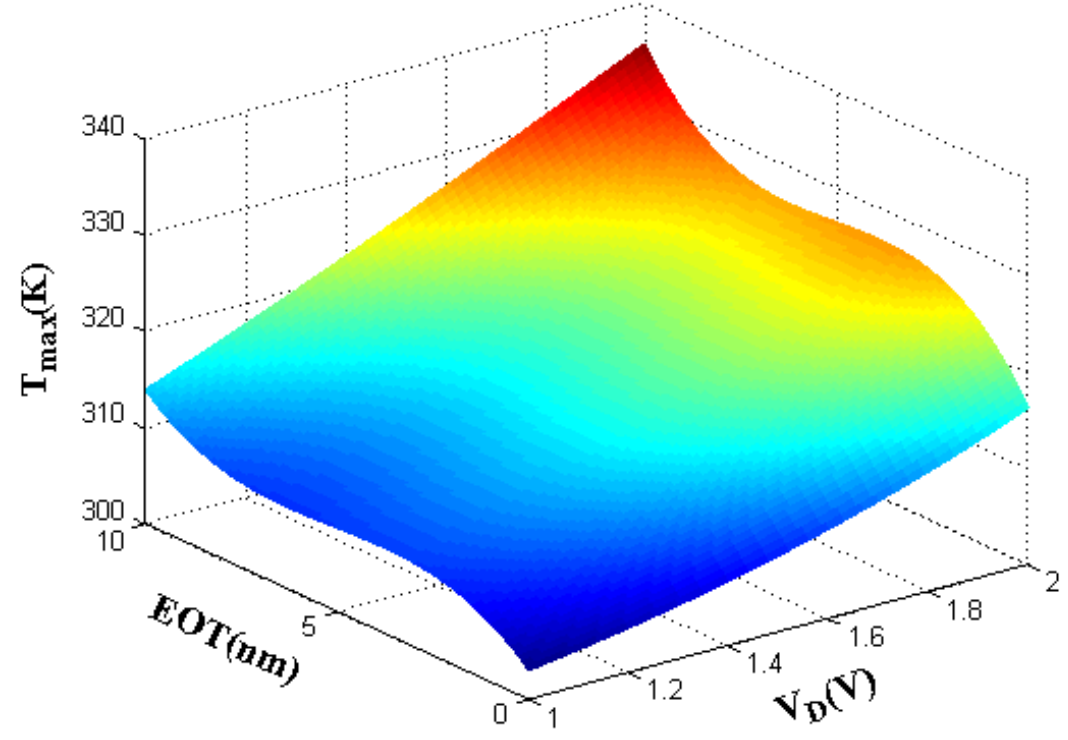


Figure 6

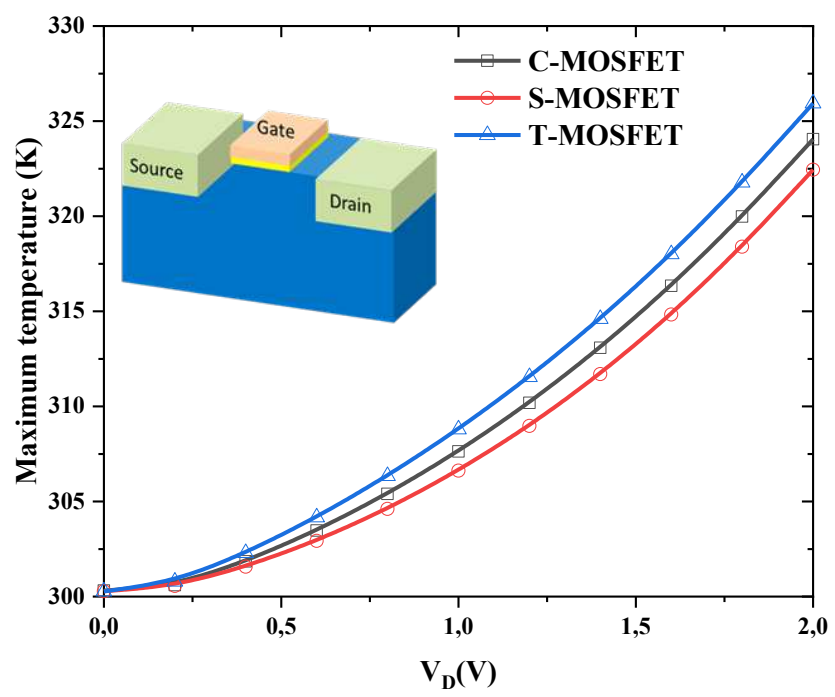


Figure 7

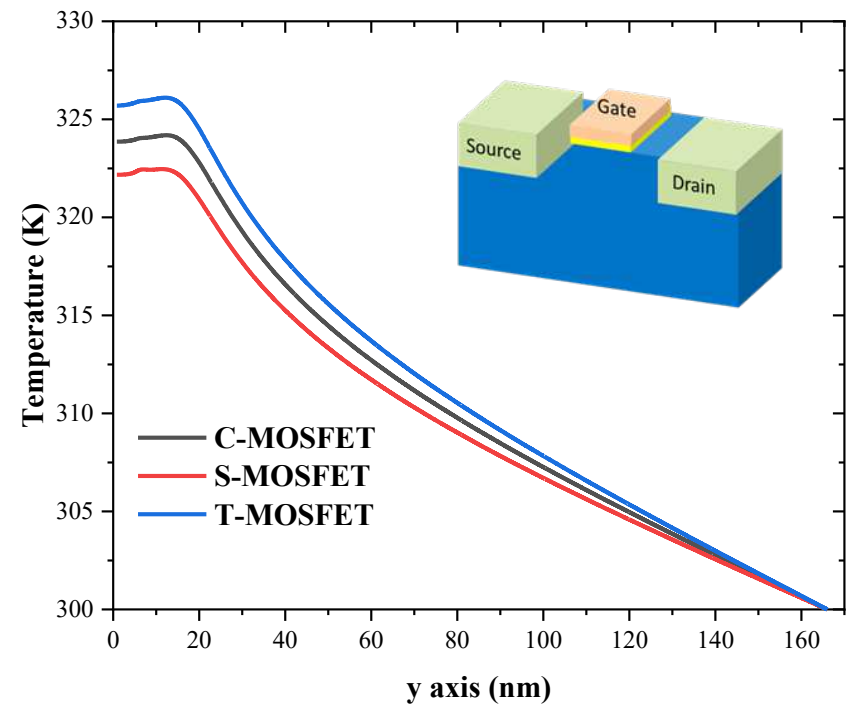


Figure 8

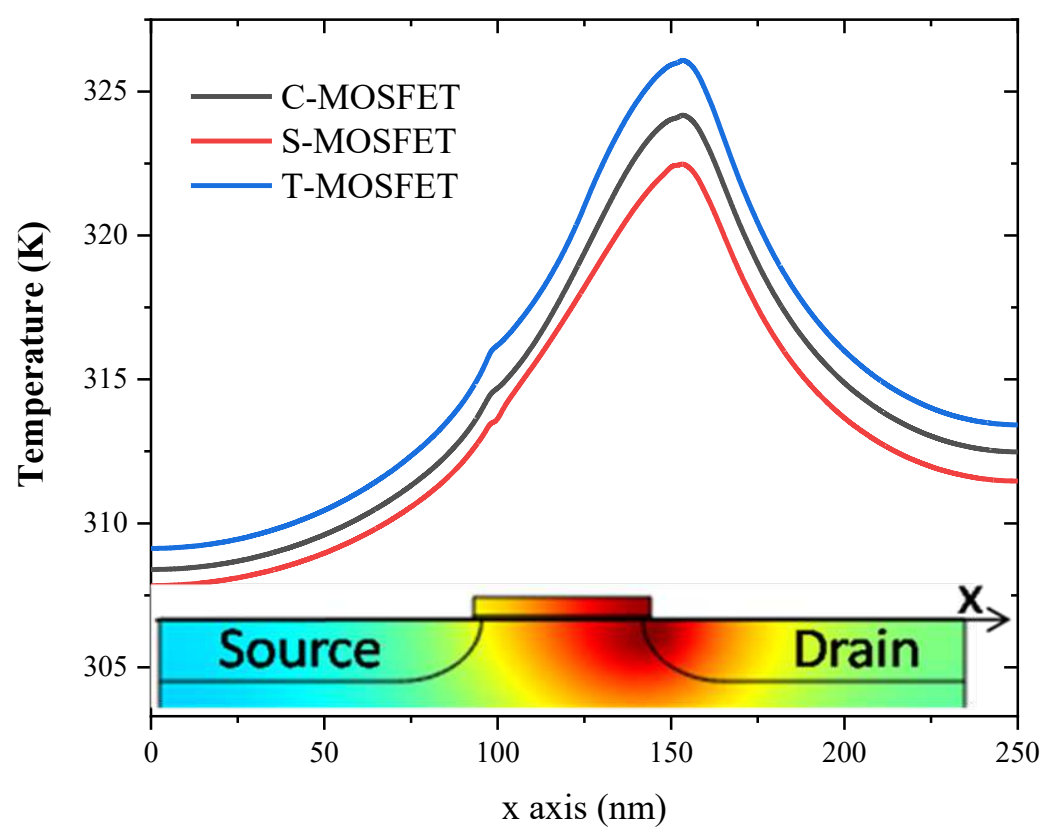


Figure 9

(a)

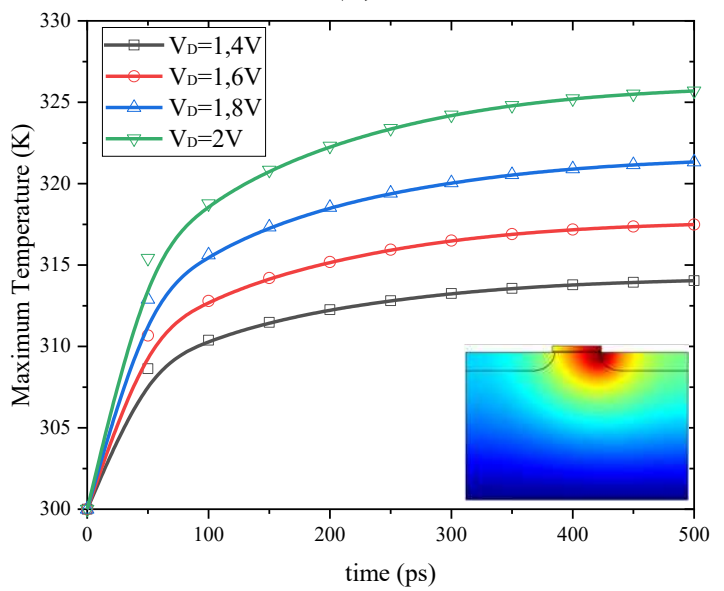

(c)

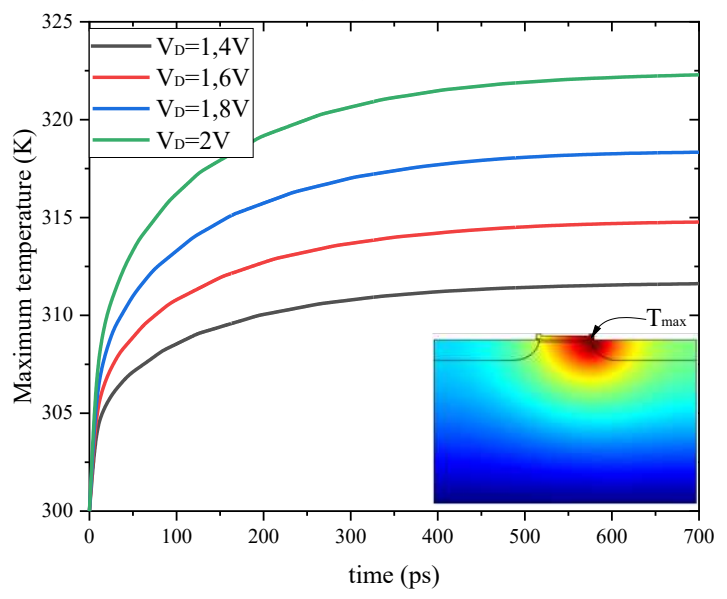

(b)

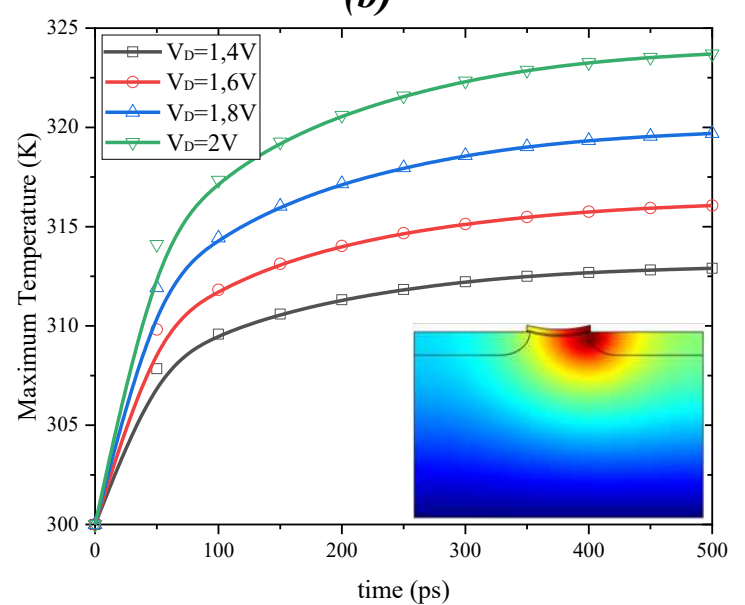

(d)

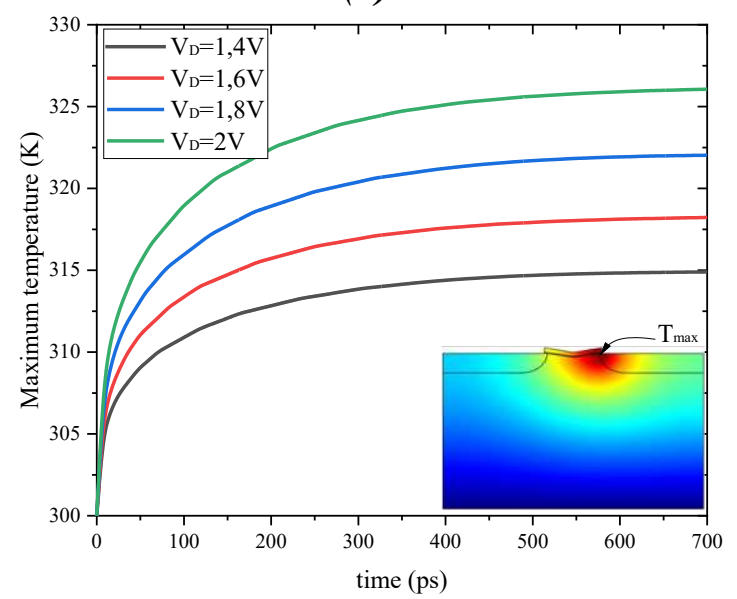


Figure 10

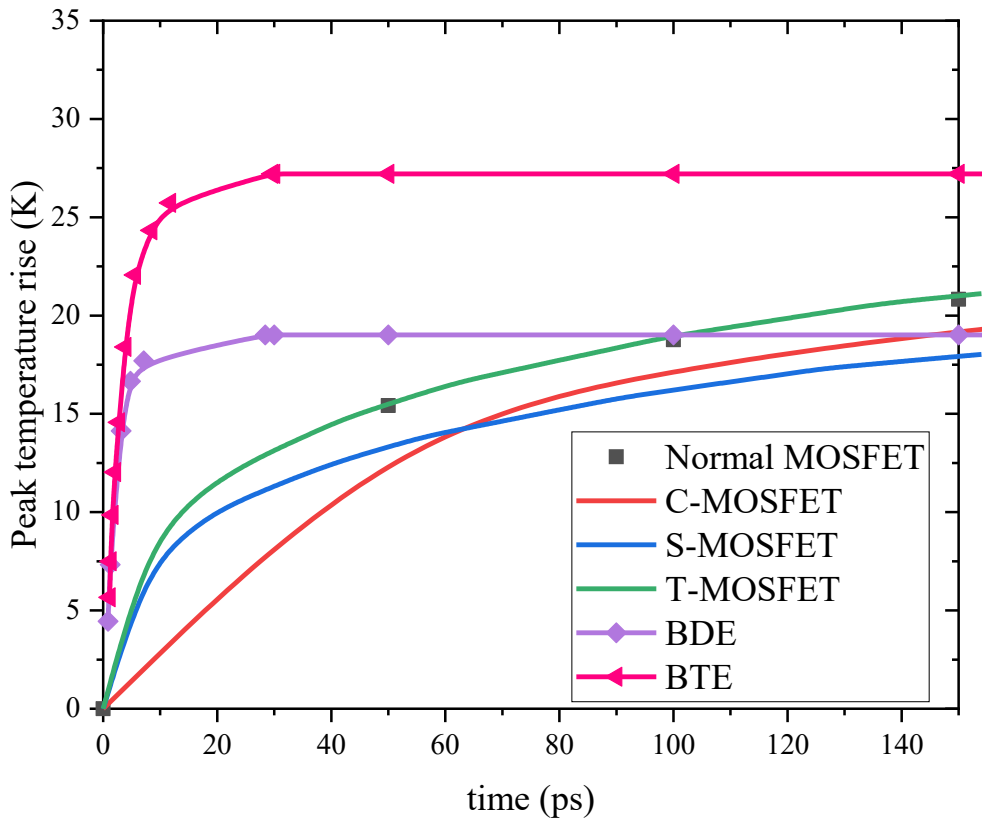




\section{Table 1}

\begin{tabular}{ll}
\hline Parameter & Value \\
\hline Channel length & $50 \mathrm{~nm}$ \\
Substrate length & $250 \mathrm{~nm}$ \\
Vertical spacing of the device & $160 \mathrm{~nm}$ \\
oxide thickness & $1 \mathrm{~nm}$ \\
doping concentration in Source /drain & $1 \times 10^{20} \mathrm{~cm}^{-3}$ \\
Channel doping concentration & $1 \times 10^{17} \mathrm{~cm}^{-3}$ \\
\hline
\end{tabular}




\section{Figures}

(a)

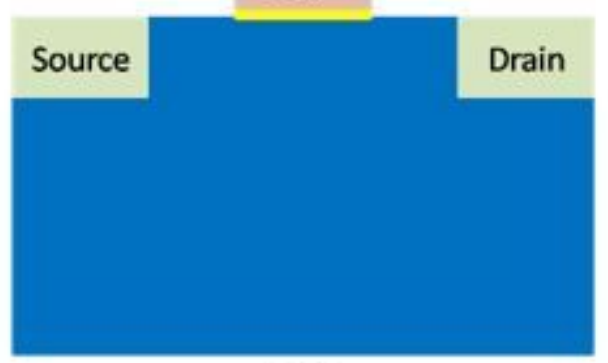

(c)

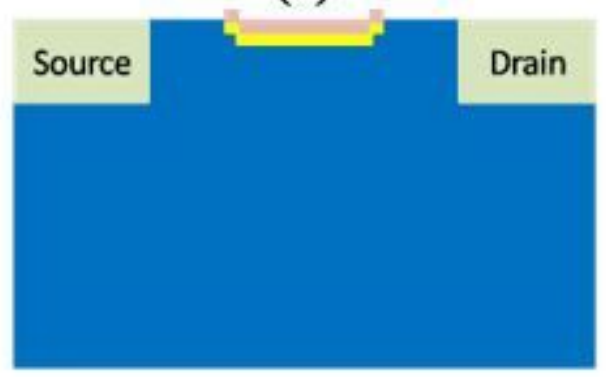

(b)

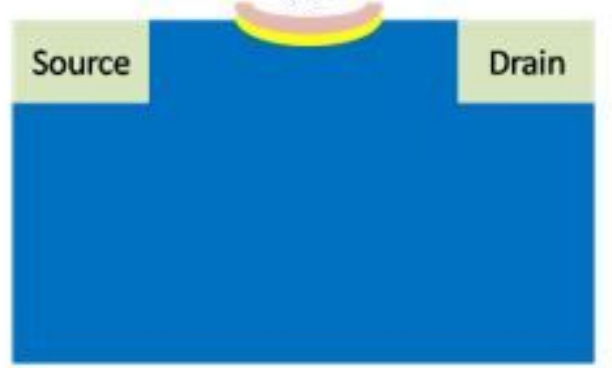

(d)

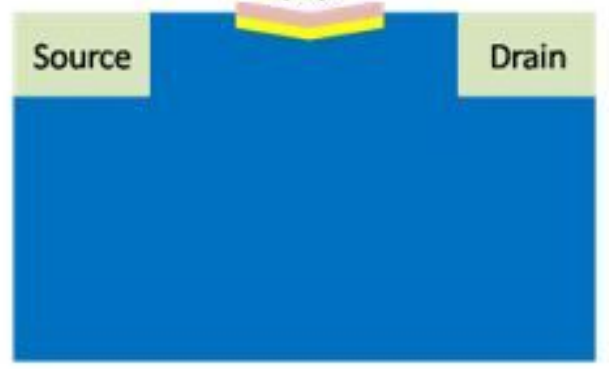

\section{Figure 1}

2D Normal MOSFET (a), MOSFET with curve gate shape (b), MOSFET with square gate shape (c) and MOSFET with triangular gate shape (d)

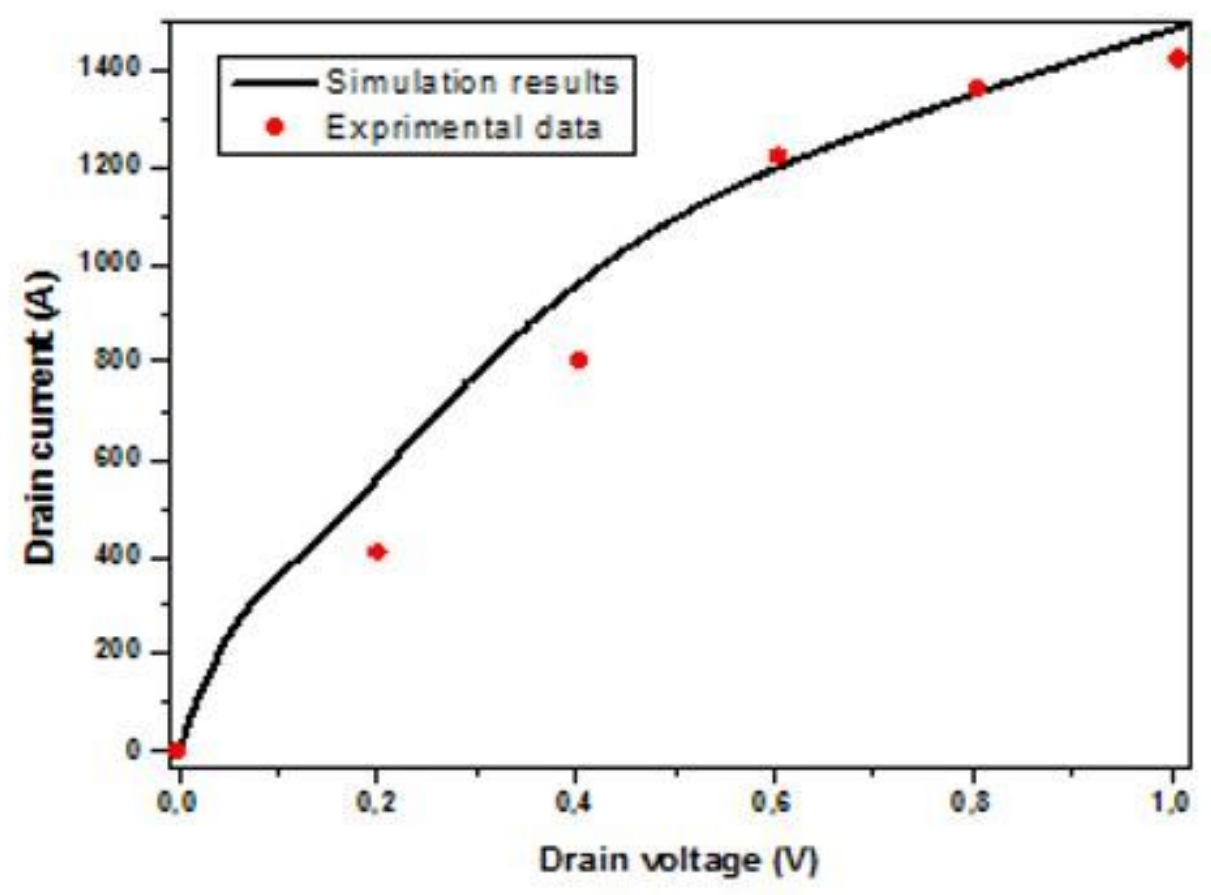

Figure 2 
Comparison of the output caractesistics of simulated results with exprimental data[21]

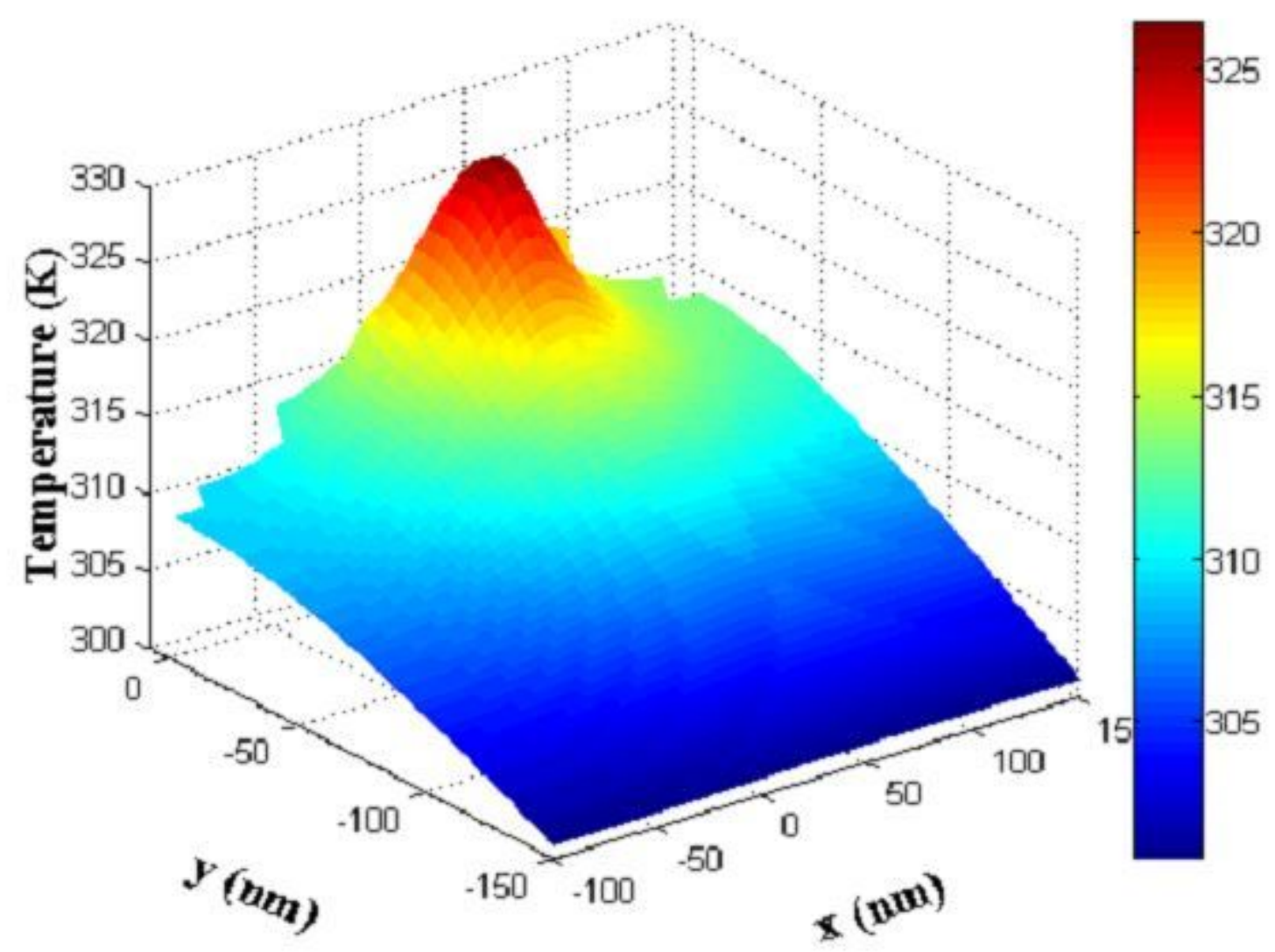

Figure 3

Temperature distribution along X-Y plane 


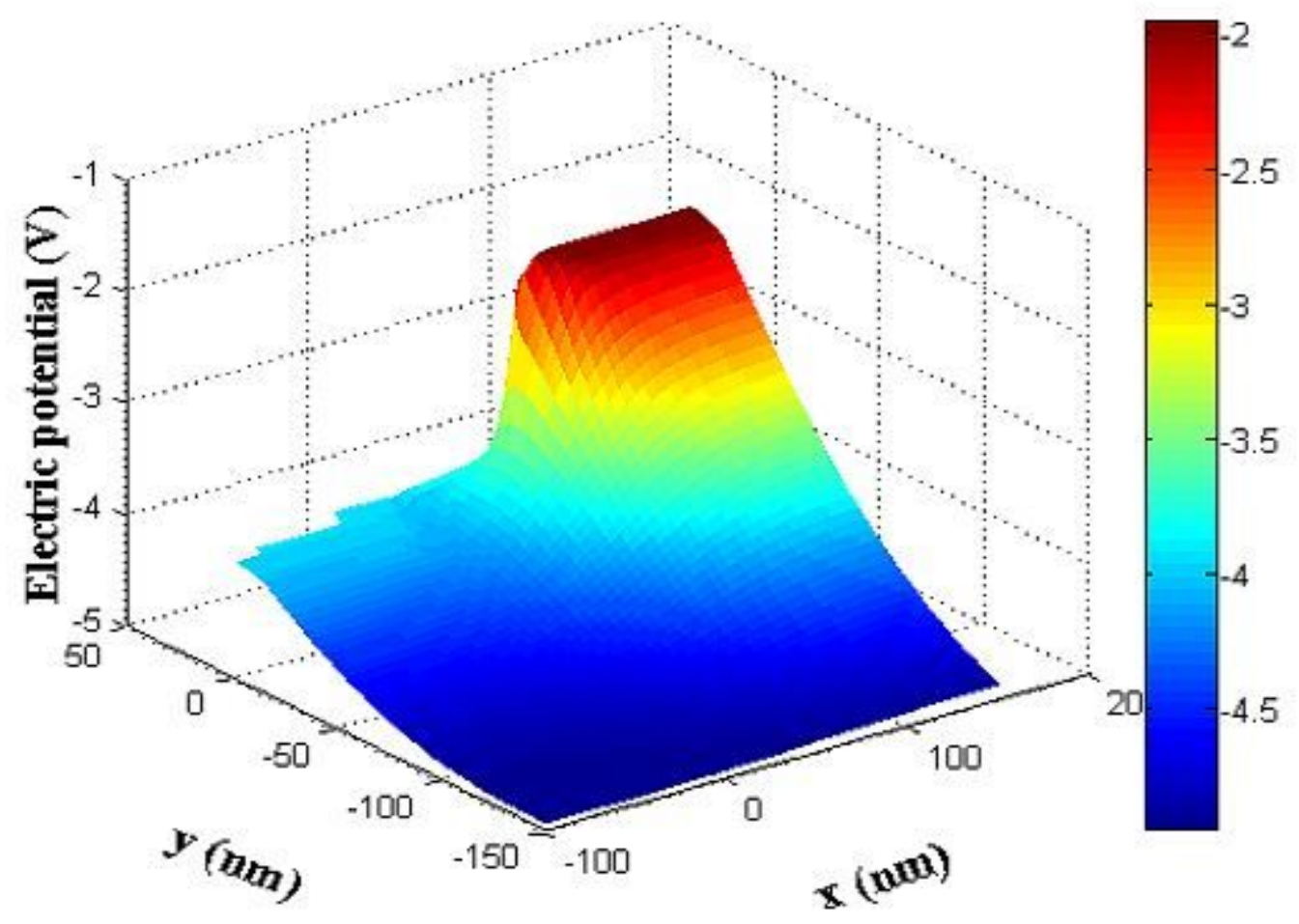

Figure 4

Electric potential distribution along X-Y plane 


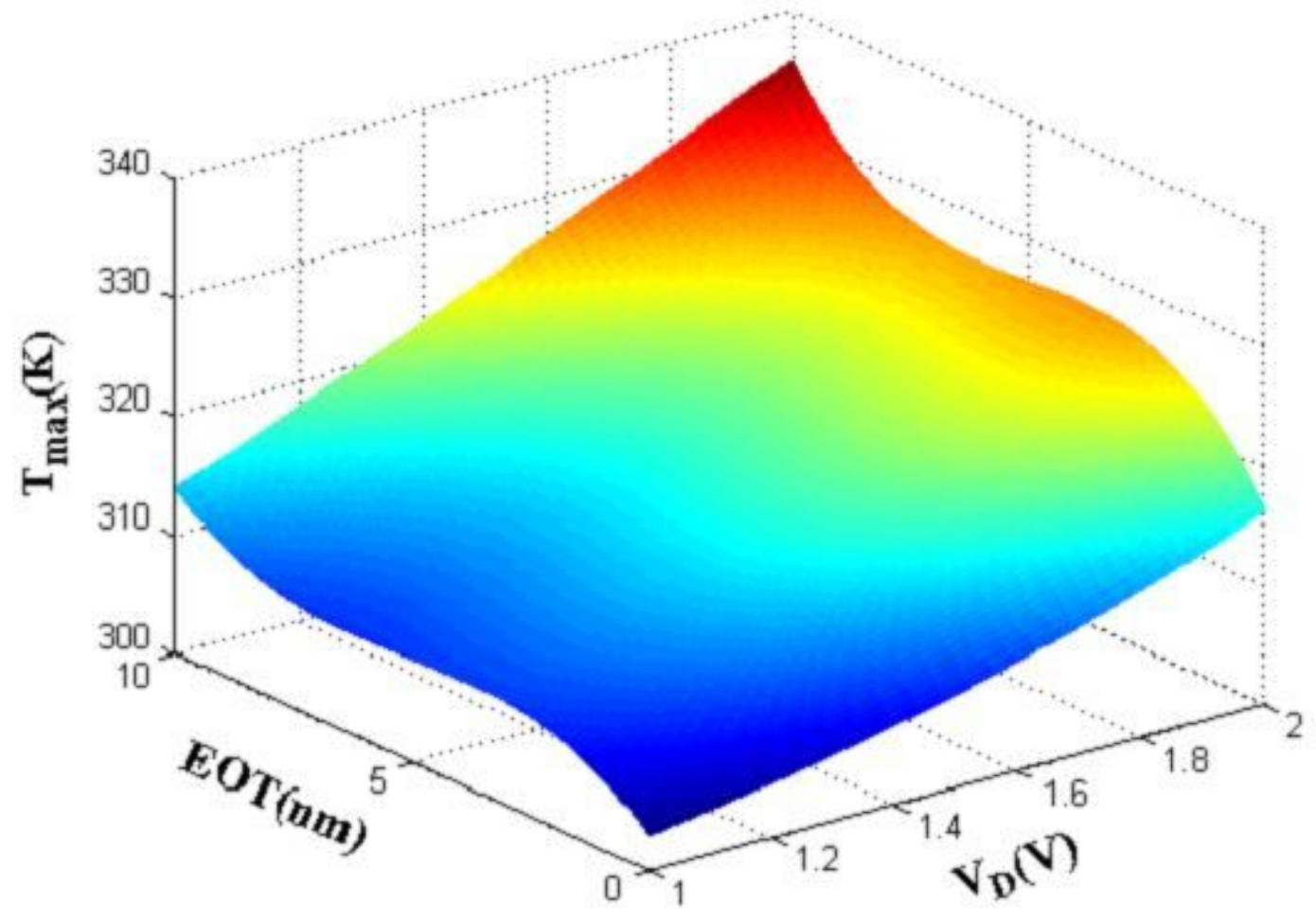

Figure 5

Temperature distribution for different EOT and variable drain voltage

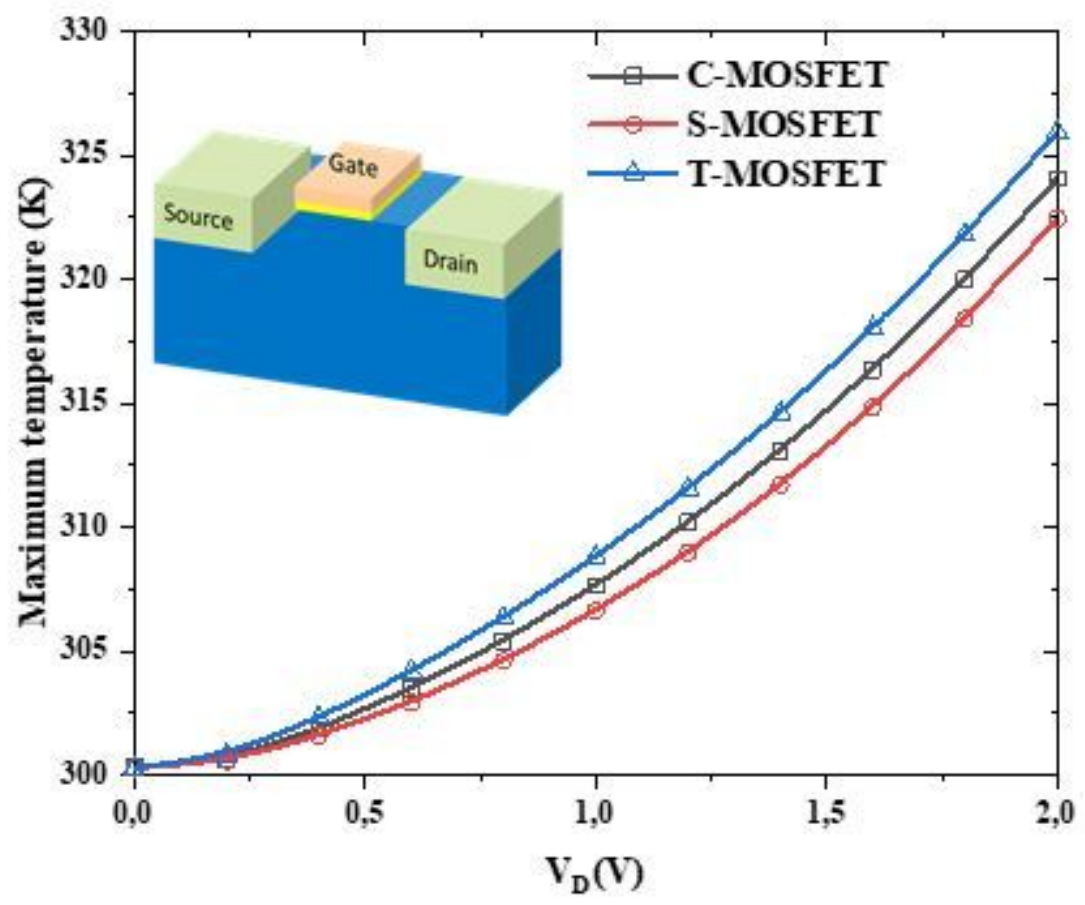


Figure 6

Maximum temperature profile versus drain voltage in MOSFET structure with different gate shape

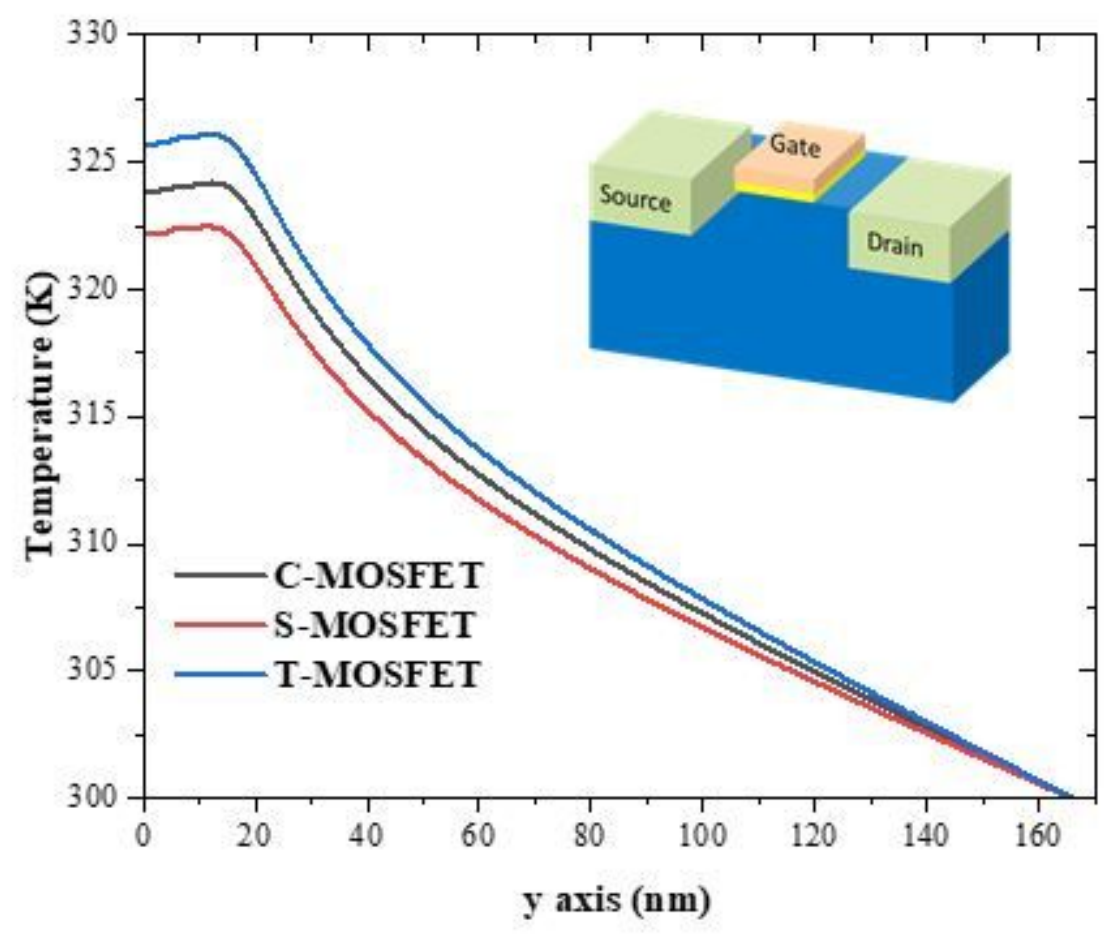

Figure 7

Temperature profile along the $y$ axis in MOSFET structure with different gate shape 


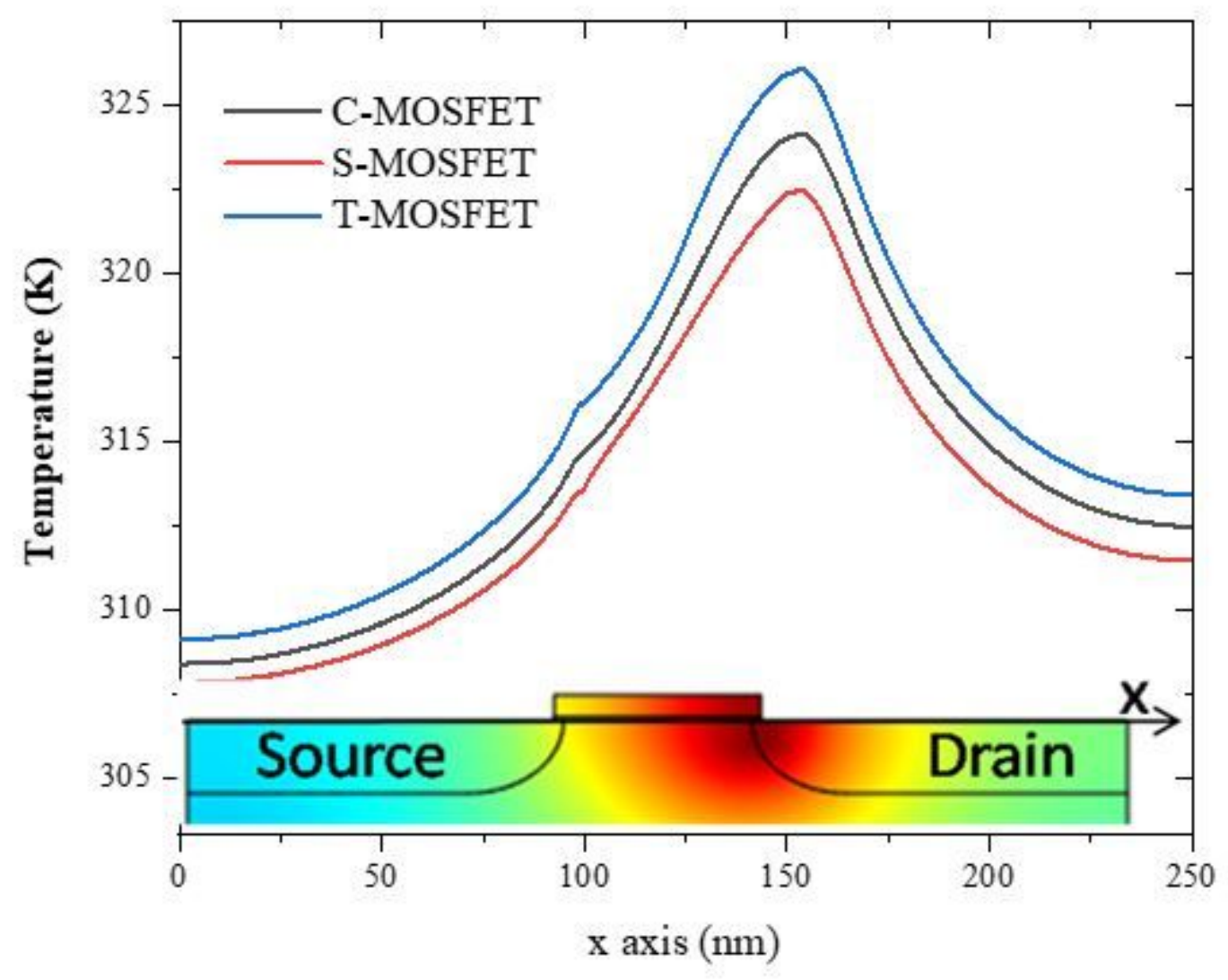

Figure 8

Temperature profile along the $x$ axis in MOSFET structure with different gate shape 
(a)

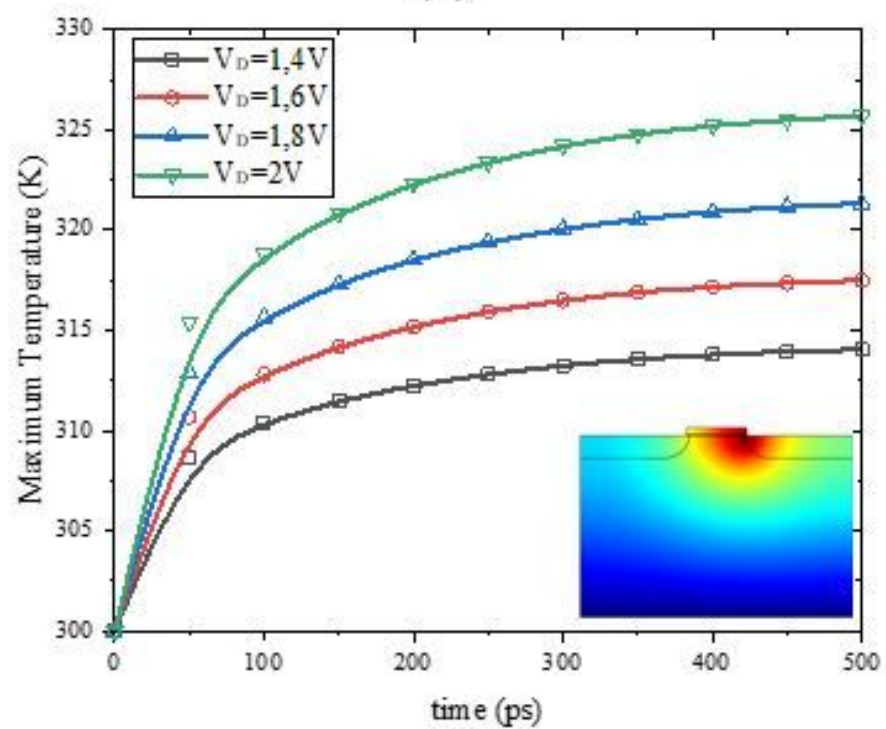

(c)

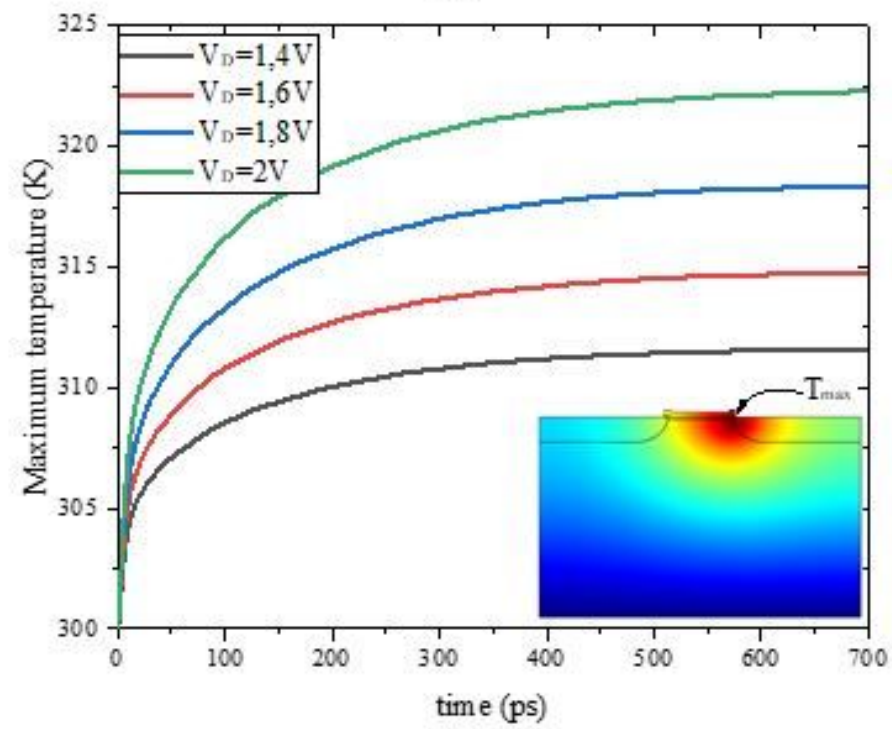

(b)

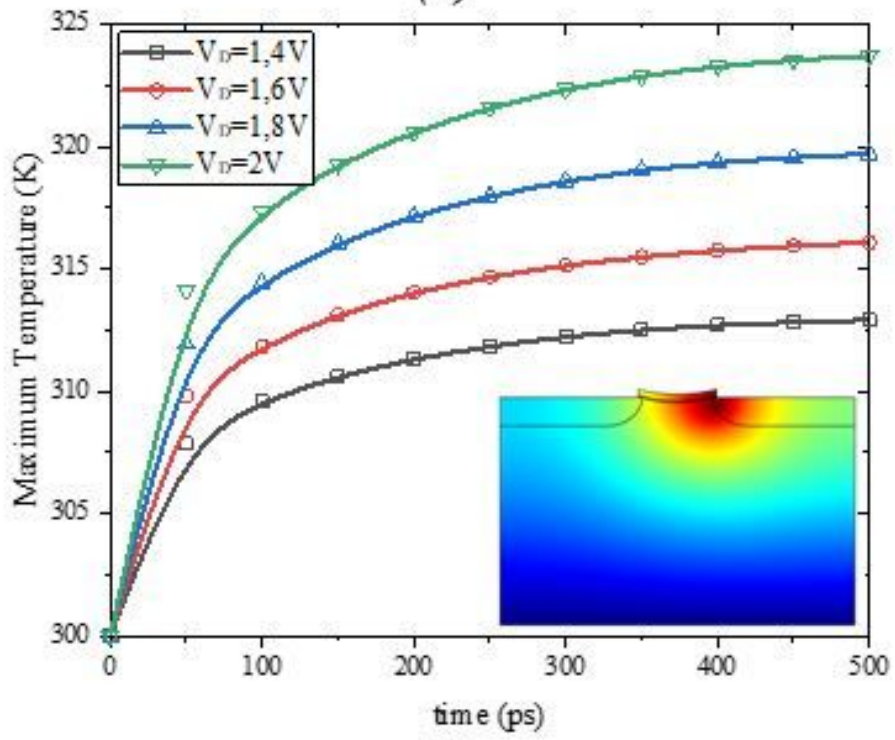

(d)

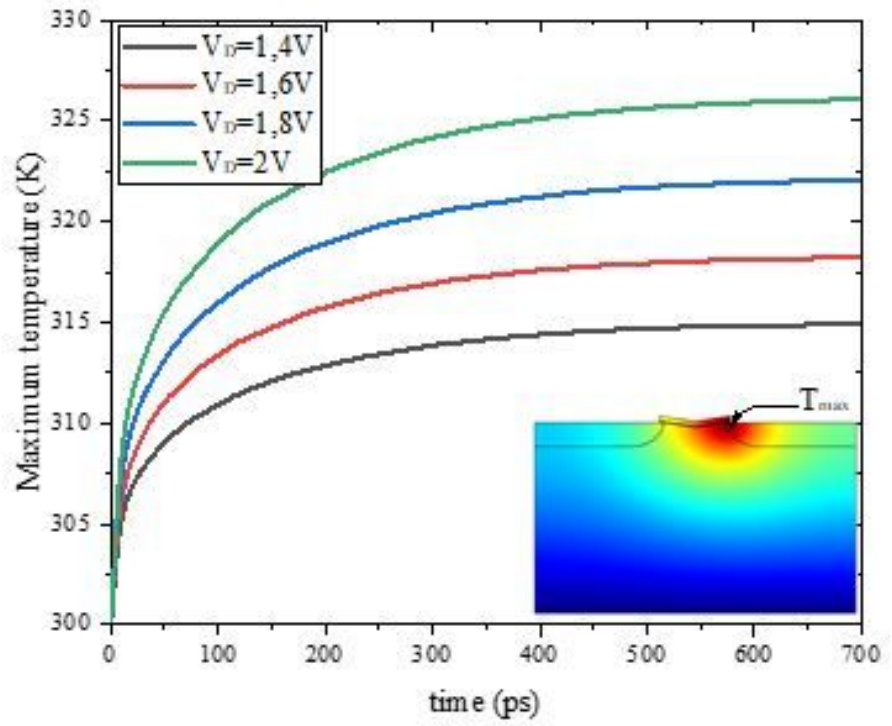

\section{Figure 9}

Transient temperature profile in MOSFETs for different drain voltages. 


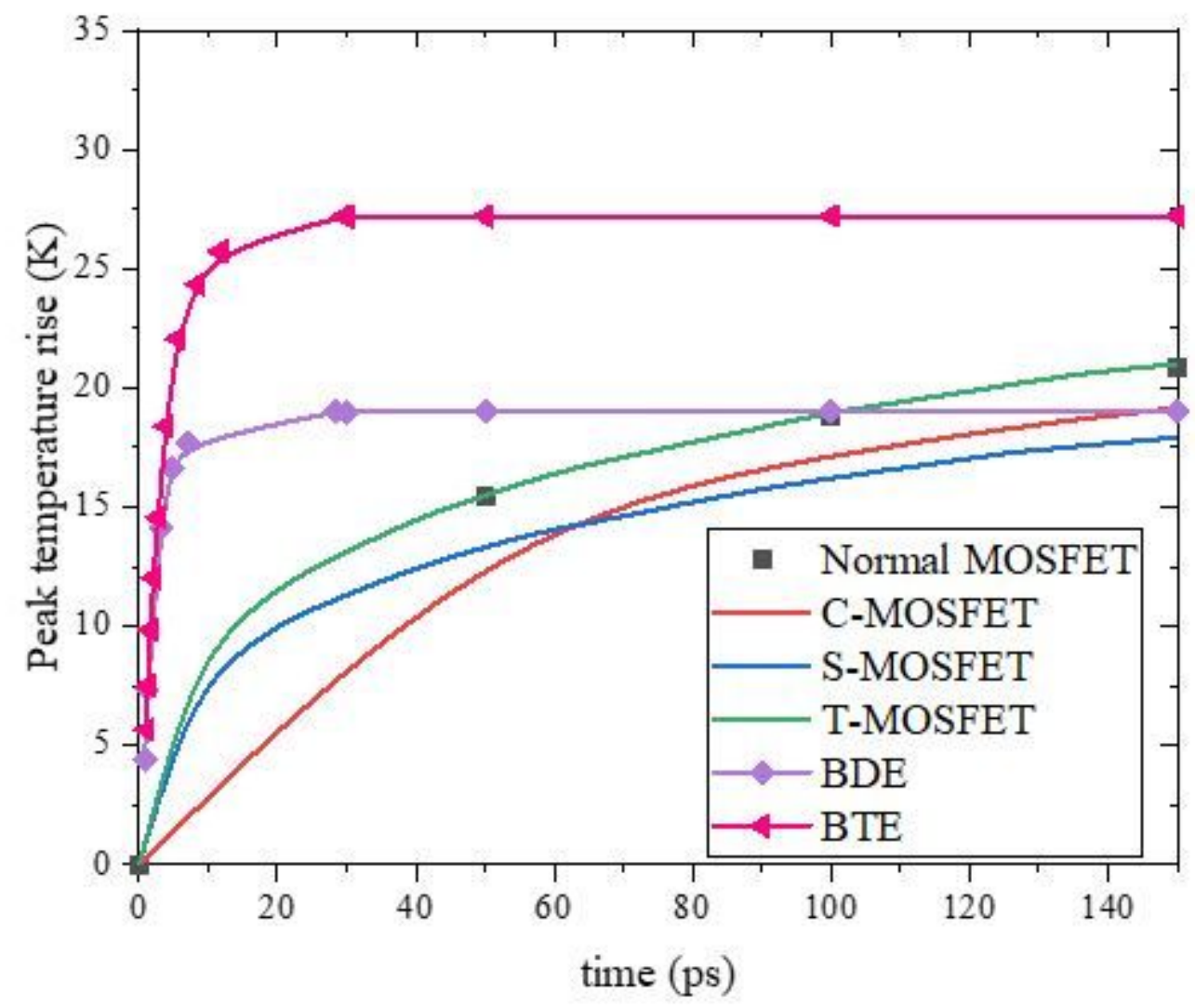

Figure 10

Comparaison of Transient temperature profile in different structures 